\title{
CORRECTOR-PREDICTOR METHODS FOR SUFFICIENT LINEAR COMPLEMENTARITY PROBLEMS IN A WIDE NEIGHBORHOOD OF THE CENTRAL PATH
}

\author{
XING LIU* AND FLORIAN A. POTRA ${ }^{\dagger}$
}

Abbreviated title: Corrector-predictor methods for sufficient LCP

Abstract. A higher order corrector-predictor interior-point method is proposed for solving sufficient linear complementarity problems. The algorithm produces a sequence of iterates in the $\mathcal{N}_{\infty}^{-}$ neighborhood of the central path. The algorithm does not depend on the handicap $\kappa$ of the problem. It has $O((1+\kappa) \sqrt{n} L)$ iteration complexity and is superlinearly convergent even for degenerate problems.

Key words. linear complementarity, interior-point, path-following, corrector-predictor, wide neighborhood

AMS subject classifications. 90C51, 90C 33

1. Introduction. The MTY predictor-corrector algorithm proposed by Mizuno, Todd and Ye [9] is a typical representative of a large class of MTY type predictorcorrector methods, which play a very important role among primal-dual interior point methods. It was the first algorithm for linear programming (LP) that had both polynomial complexity and superlinear convergence. This result was extended to monotone linear complementarity problems that are nondegenerate, in the sense that they have a strictly complementarity solution $[6,23]$. It turned out that the nondegeneracy assumption is not restrictive, since according to [10] a large class of interior point methods, which contains MTY, can have only linear convergence if this assumption is violated. However, it is possible to obtain arbitrarily high order of convergence for degenerate problems by using higher order information of the central path [19, 21].

The existence of a central path is crucial for interior-point methods. An important result of the 1991 monograph of Kojima et al. [7] shows that the central path exists for any $P_{*}$ linear complementarity problem, provided that the relative interior of its feasible set is nonempty. We recall that every $P_{*}$ linear complementarity problem is a $P_{*}(\kappa)$ problem for some $\kappa \geq 0$, i.e.

$$
P_{*}=\cup_{\kappa \geq 0} P_{*}(\kappa) .
$$

The class of sufficient matrices was introduced by Cottle et al. [3] in connection with the linear complementarity problems. A matrix $M \in R^{n \times n}$ is said to be column sufficient if:

$$
z_{i}(M z)_{i} \leq 0 \text { for all } i \text { implies } z_{i}(M z)_{i}=0 \text { for all } i .
$$

The matrix $M$ is called row sufficient if its transpose is column sufficient. The matrix $M$ is sufficient if it is both column and row sufficient. It is proved in the same book that the class of sufficient matrices is closely related to the existence of the solution of the linear complementarity problems and the convexity of the solution set. A surprising result given by Väliaho [22] showed that the class of $P_{*}$ matrices coincides with the class of sufficient matrices. Therefore, every $P_{*}$ linear complementarity problem

\footnotetext{
*Department of Mathematics and Statistics, University of Maryland Baltimore County.
}

${ }^{\dagger}$ Department of Mathematics and Statistics, University of Maryland Baltimore County. 
is a sufficient linear complementarity problem, and vice versa. The class of sufficient linear complementarity problems is a very general framework for studying interiorpoint methods. In 1995 Miao [8] extended the MTY predictor-corrector algorithm for $P_{*}(\kappa)$ linear complementarity problems. His algorithm has $O((1+\kappa) \sqrt{n} L)$ iteration complexity, and is quadratically convergent for nondegenerate problems. However, the constant $\kappa$ is explicitly used in the construction of the algorithm, which implies that the algorithm cannot be used for sufficient linear complementarity problems. Potra and Sheng [17] extended the MTY predictor-corrector algorithm further for sufficient complementarity problems. While the algorithms of [17] do not depend on the constant $\kappa$, their computational complexity does: if the problem is a $P_{*}(\kappa)$ linear complementarity problem they terminate in at most $O((1+\kappa) \sqrt{n} L)$ iterations. Moreover, the algorithms may attain arbitrarily high orders of convergence on nondegenerate problems. Predictor-corrector algorithms with arbitrarily high order of convergence for degenerate sufficient linear complementarity problems were given in [19]. The algorithms, as shown in [18], have $O((1+\kappa) \sqrt{n} L)$ iteration complexity for $P_{*}(\kappa)$ linear complementarity problems.

All the above algorithms operate in $l_{2}$ neighborhoods, also known as the small neighborhoods, of the central path. It is well known however that primal-dual interiorpoint methods have better practical performances in wide neighborhoods of the central path. Unfortunately, the iteration complexity of the predictor-corrector methods that use wide neighborhoods are worse than the complexity of the corresponding methods for small neighborhoods. Moreover, as shown in $[2,4]$, it is more difficult to develop and analyze predictor-corrector methods in wide neighborhoods. The best iteration complexity achieved by any known interior-point method for monotone linear complementarity problems in the wide neighborhoods using first order information is $O(n L)$. By using a large neighborhood defined by a suitable self regular proximity measure, Peng et al. [12] have obtained a predictor-corrector method with $O(\log n \sqrt{n} L)$ iteration complexity which is superlinearly convergent on nondegenerate problems. It turns out that the complexity result can be improved by using higher order information. The algorithms described in $[11,5,24]$ have $O(\sqrt{n} L)$ iteration complexity. However, these algorithms are not of a predictor-corrector type, and they are not superlinearly convergent. The algorithm described in [20] operates in the $\delta_{\infty}^{-}$neighborhood, and is superlinear convergent for sufficient linear complementarity problems, but no complexity results have been proved for this algorithm. A predictor-corrector method for monotone linear complementarity problems using wide neighborhoods of the central path was proposed in [14]. The algorithm has $O(\sqrt{n} L)$ iteration complexity by using a higher order predictor, and it is superlinear convergence even for degenerate problems. In a recent paper, Potra and Liu [16] extended the algorithm in [14] to sufficient linear complementarity problems. Two algorithms are analyzed in [16]. Both algorithms are of predictor-corrector type acting in between two wide neighborhoods of the central path. The radii of those neighborhoods have to satisfy an inequality that depends on the handicap $\kappa$ of the problems. The first algorithm in [16] depends also on $\kappa$, while the second does not. The second algorithm uses the first algorithm by assigning $\kappa=1$ and then doubles $\kappa$ until a certain criterion is satisfied. Both algorithms have $O\left((1+\kappa)^{1+1 / m} \sqrt{n} L\right)$ iteration complexity and are superlinearly convergent even for degenerate problems.

The traditional predictor-corrector algorithms operate between two neighborhoods of the central path. The predictor step aims to decrease the duality gap while keeping the point in the outer neighborhood. It is followed by a corrector step, which 
brings the point back into the inner neighborhood so that the next predictor-corrector iteration can be performed. As analyzed in a recent paper [15], the centering direction is not as efficient in the wide neighborhoods as in the small neighborhoods, so that a line search on the centering direction is always needed in the corrector step using wide neighborhoods. Moreover, since the pure centering direction is anyhow inefficient in the wide neighborhoods, a corrector-predictor method was proposed in [15] where the corrector is used to improve both optimality and centrality. In the present paper, we generalize this algorithm to sufficient linear complementarity problems. By using higher order information, the algorithm has $O((1+\kappa) \sqrt{n} L)$ iteration complexity, which matches the best iteration complexity obtained in the small neighborhoods. Moreover, our algorithm is superlinearly covergent even for degenerate problems. More precisely, by using a predictor with order $m_{p}>1$, we show that the duality gap converges to zero with Q-order $m_{p}+1$ in the nondegenerate case, and with Q-order $\left(m_{p}+1\right) / 2$ in the degenerate case. Our algorithm improves considerably the results of [16]. First, the algorithm is a corrector-predictor interior-point method so that it uses only one wide neighborhood of the central path, whose radius can be any number between 0 and 1 , and therefore does not depend on $\kappa$. Second its iteration complexity is improved $\left(O((1+\kappa) \sqrt{n} L)\right.$ versus $\left.O\left((1+\kappa)^{1+1 / m} \sqrt{n} L\right)\right)$. Finally, by contrast with the algorithms of [16] the present algorithm reduces the duality gap both in the corrector and the predictor steps and therefore it is more efficient. In the present paper we work on horizontal linear complementarity problems (HLCP), which is a slight generalization of the standard linear complementarity problem. Equivalence results of different variants of linear complementarity problems can be found in [1]. We choose to work on HLCP because of its symmetry.

We denote by $\mathbb{N}$ the set of all nonnegative integers. $\mathbb{R}, \mathbb{R}_{+}, \mathbb{R}_{++}$denote the set of real, nonnegative real, and positive real numbers respectively. For any real number $\kappa,\lceil\kappa\rceil$ denotes the smallest integer greater or equal to $\kappa$. Given a vector $x$, the corresponding upper case symbol denotes, as usual, the diagonal matrix $X$ defined by the vector. The symbol $e$ represents the vector of all ones, with dimension given by the context.

We denote component-wise operations on vectors by the usual notations for real numbers. Thus, given two vectors $u, v$ of the same dimension, $u v, u / v$, etc. will denote the vectors with components $u_{i} v_{i}, u_{i} / v_{i}$, etc. This notation is consistent as long as component-wise operations always have precedence in relation to matrix operations. Note that $u v \equiv U v$ and if $A$ is a matrix, then $A u v \equiv A U v$, but in general $A u v \neq(A u) v$. Also if $f$ is a scalar function and $v$ is a vector, then $f(v)$ denotes the vector with components $f\left(v_{i}\right)$. For example if $v \in \mathbb{R}_{+}^{n}$, then $\sqrt{v}$ denotes the vector with components $\sqrt{v_{i}}$, and $1-v$ denotes the vector with components $1-v_{i}$. Traditionally the vector $1-v$ is written as $e-v$, where $e$ is the vector of all ones. Inequalities are to be understood in a similar fashion. For example if $v \in \mathbb{R}^{n}$, then $v \geq 3$ means that $v_{i} \geq 3, i=1, \ldots, n$. Traditionally this is written as $v \geq 3 e$. If $\|$. is a vector norm on $\mathbb{R}^{n}$ and $A$ is a matrix, then the operator norm induced by $\|\cdot\|$ is defined by $\|A\|=\max \{\|A x\| ;\|x\|=1\}$. As a particular case we note that if $U$ is the diagonal matrix defined by the vector $u$, then $\|U\|_{2}=\|u\|_{\infty}$.

We use the notations $O(\cdot), \Omega(\cdot), \Theta(\cdot)$, and $o(\cdot)$ in the standard way to express asymptotic relationships between functions. The most common usage will be associated with a sequence $\left\{x^{k}\right\}$ of vectors and a sequence $\left\{\tau_{k}\right\}$ of positive real numbers. In this case $x^{k}=O\left(\tau_{k}\right)$ means that there is a constant $K$ (dependent on problem data) such that for every $k \in \mathbb{N},\left\|x^{k}\right\| \leq K \tau_{k}$. Similarly, if $x^{k}>0, x^{k}=\Omega\left(\tau_{k}\right)$ means that 
$\left(x^{k}\right)^{-1}=O\left(1 / \tau_{k}\right)$. If we have both $x^{k}=O\left(\tau_{k}\right)$ and $x^{k}=\Omega\left(\tau_{k}\right)$, we write $x^{k}=\Theta\left(\tau_{k}\right)$

If $x, s \in \mathbb{R}^{n}$, then the vector $z \in \mathbb{R}^{2 n}$ obtained by concatenating $x$ and $s$ will be denoted by $\lceil x, s\rfloor$, i.e.,

$$
z=\lceil x, s\rfloor=\left[\begin{array}{c}
x \\
s
\end{array}\right]=\left[x^{T}, s^{T}\right]^{T} .
$$

Throughout this paper the mean value of $x s$ will be denoted by

$$
\mu(z)=\frac{x^{T} s}{n} .
$$

2. The $P_{*}(\kappa)$ horizontal linear complementarity problem. Given two matrices $Q, R \in \mathbb{R}^{n \times n}$, and a vector $b \in \mathbb{R}^{n}$, the horizontal linear complementarity problem (HLCP) consists in finding a pair of vectors $z=\lceil x, s\rfloor$ such that

$$
\begin{aligned}
x s & =0 \\
Q x+R s & =b \\
x, s & \geq 0 .
\end{aligned}
$$

The standard (monotone) linear complementarity problem (SLCP or simply LCP) is obtained by taking $R=-I$, and $Q$ positive semidefinite. Let $\kappa \geq 0$ be a given constant. We say that $(2.1)$ is a $P_{*}(\kappa)$ HLCP if

$$
Q u+R v=0 \text { implies }(1+4 \kappa) \sum_{i \in \mathcal{I}^{+}} u_{i} v_{i}+\sum_{i \in \mathcal{I}^{-}} u_{i} v_{i} \geq 0, \text { for any } u, v \in \mathbb{R}^{n},
$$

where $\mathcal{I}^{+}=\left\{i: u_{i} v_{i}>0\right\}$ and $\mathcal{I}^{-}=\left\{i: u_{i} v_{i}<0\right\}$. If the above condition is satisfied we say that $(Q, R)$ is a $P_{*}(\kappa)$ pair and we write $(Q, R) \in P_{*}(\kappa)$. In case $R=-I$, $(Q,-I)$ is a $P_{*}(\kappa)$ pair if and only if $Q$ is a $P_{*}(\kappa)$ matrix in the sense that:

$$
(1+4 \kappa) \sum_{i \in \hat{\mathcal{I}}^{+}} x_{i}[Q x]_{i}+\sum_{i \in \hat{\mathcal{I}}^{-}} x_{i}[Q x]_{i} \geq 0, \quad \forall x \in \mathbb{R}^{n},
$$

where $\hat{\mathcal{I}}^{+}=\left\{i: x_{i}[Q x]_{i}>0\right\}$ and $\hat{\mathcal{I}}^{-}=\left\{i: x_{i}[Q x]_{i}<0\right\}$. Problem (2.1) is then called a $P_{*}(\kappa) \mathrm{LCP}$ and it is extensively discussed in [7]. If $(Q, R)$ belongs to the class

$$
P_{*}=\cup_{\kappa \geq 0} P_{*}(\kappa),
$$

then we say that $(Q, R)$ is a $P_{*}$ pair and $(2.1)$ is a $P_{*}$ HLCP.

The class of sufficient matrices was defined by Cottle et al. in [3]. The appropriate generalization to sufficient pair $[18,19]$ is in terms of the null space of the matrix $[Q R] \in \mathbb{R}^{n \times 2 n}$

$$
\Phi:=\mathcal{N}([Q R])=\{\lceil u, v\rfloor \mid Q u+R v=0\}
$$

and its orthogonal space

$$
\Phi^{\perp}=\left\{\lceil u, v\rfloor \mid u=Q^{T} x, v=R^{T} x, \text { for some } x \in \mathbb{R}^{n}\right\} .
$$

$(Q, R)$ is called column sufficient if

$$
\lceil u, v\rfloor \in \Phi, \quad u v \leq 0 \text { implies } u v=0,
$$


and row sufficient if

$$
\lceil u, v\rfloor \in \Phi^{\perp}, \quad u v \geq 0 \text { implies } u v=0 .
$$

$(Q, R)$ is a sufficient pair if it is both column and row sufficient. The corresponding results of row and column sufficient matrices in [3] can be extended to row and column sufficient pairs (see, for example, $[20]):(Q, R)$ is a sufficient pair if and only if for any $b$, the HLCP (2.1) has a convex (perhaps empty) solution set and every KKT point of

$$
\begin{array}{cc}
\min _{x, s} & x^{T} s \\
\text { s.t. } & Q x+R s=b \\
& x, s \geq 0
\end{array}
$$

is a solution of $(2.1)$.

Väliaho's result [22] states that a matrix is sufficient if and only if it is a $P_{*}(\kappa)$ matrix for some $\kappa \geq 0$. The result can be extended to sufficient pairs by using the equivalence results from [1] (see also $[20]):(Q, R)$ is a sufficient pair if and only if there is a finite $\kappa \geq 0$ so that $(Q, R)$ is a $P_{*}(\kappa)$ pair. By extension, a $P_{*}$ HLCP will be called a sufficient HLCP and a $P_{*}$ pair will be called a sufficient pair.

Let us note that if $(Q, R)$ is a sufficient pair, then the matrix $[Q R]$ is full rank. In fact, we have the following slightly stronger result.

TheOREM 2.1. Given two matrices $Q, R \in \mathbb{R}^{n \times n}$, if the pair $(Q, R)$ is column sufficient, the matrix $[Q R]$ is full rank.

Proof. Let $r$ be the rank of $Q$, the LU factorization of $Q$ can be written as

$$
P Q=L\left[\begin{array}{cc}
F_{1} & F_{2} \\
0 & 0
\end{array}\right]
$$

where $P$ is a permutation matrix, $L \in \mathbb{R}^{n \times n}$ is an invertible lower triangular matrix, $F_{1} \in \mathbb{R}^{n-r \times n-r}$ is an invertible upper triangular matrix, and $F_{2}$ and the zeros are matrices with the correct dimensions.

Let us denote by $G$

$$
G=L^{-1} P R=\left[\begin{array}{ll}
G_{11} & G_{12} \\
G_{21} & G_{22}
\end{array}\right]
$$

where $G_{11} \in \mathbb{R}^{n-r \times n-r}$, and $G_{12}, G_{21}, G_{22}$ are with the correct dimensions.

Since permutation matrices are invertible, and $L$ is invertible, we have

$$
\operatorname{rank}\left(\left[\begin{array}{ll}
Q & R
\end{array}\right]\right)=\operatorname{rank}\left(L^{-1} P[Q \quad R]\right)=\operatorname{rank}\left(\left[\begin{array}{cccc}
F_{1} & F_{2} & G_{11} & G_{12} \\
0 & 0 & G_{21} & G_{22}
\end{array}\right]\right) .
$$

We denote by $u_{1}$ and $u_{2}$ the components of $u$ in the first $r$ and last $n-r$ indices, respectively, and similarly for $v$. Therefore, $\lceil u, v\rfloor \in \Phi$ is equivalent to

$$
\left\{\begin{array}{r}
F_{1} u_{1}+F_{2} u_{2}+G_{11} v_{1}+G_{12} v_{2}=0 \\
G_{21} v_{1}+G_{22} v_{2}=0
\end{array} .\right.
$$

For any $v_{2} \in \operatorname{Ker}\left(G_{22}\right)$, we construct a pair of vectors $u$ and $v$ such that

$$
v_{1}=0, \quad u_{2}=-v_{2}, \quad u_{1}=-F_{1}^{-1}\left(F_{2} u_{2}+G_{12} v_{2}\right) .
$$


Clearly we have $\lceil u, v\rfloor \in \Phi$. Moreover, we also obtain

$$
u_{1} v_{1} \leq 0, \quad \text { and } \quad u_{2} v_{2}=-v_{2}^{2} \leq 0
$$

Because $(Q, R)$ is column sufficient, we have

$$
u_{1} v_{1}=0, \quad \text { and } \quad u_{2} v_{2}=0 .
$$

We thus have $v_{2} \in \operatorname{Ker}\left(G_{22}\right)$ implies $v_{2}=0$. Therefore $G_{22}$ is invertible, and

$$
\operatorname{rank}([Q \quad R])=\operatorname{rank}\left(\left[\begin{array}{cccc}
F_{1} & F_{2} & G_{11} & G_{12} \\
0 & 0 & G_{21} & G_{22}
\end{array}\right]\right)=n .
$$

It is interesting to remark that row sufficiency alone does not imply the full rank property. For example, take

$$
Q=\left[\begin{array}{ll}
1 & 0 \\
0 & 0
\end{array}\right] \quad \text { and } \quad R=\left[\begin{array}{cc}
-1 & 0 \\
0 & 0
\end{array}\right]
$$

it is easily seen that $(Q, R)$ is row sufficient, but $\operatorname{rank}([Q R])=1$. We also note that in $[19,18,20]$, the full rank property was given as an assumption, which in fact always hold because of the above theorem.

We denote the set of all feasible points of HLCP by

$$
\mathcal{F}=\left\{z=\lceil x, s\rfloor \in \mathbb{R}_{+}^{2 n}: \quad Q x+R s=b\right\},
$$

and its solution set by

$$
\mathcal{F}^{*}=\left\{z^{*}=\left\lceil x^{*}, s^{*}\right\rfloor \in \mathcal{F}: \quad x^{*} s^{*}=0\right\} .
$$

The relative interior of $\mathcal{F}$, which is also known as the set of strictly feasible points or the set of interior points, is given by

$$
\mathcal{F}^{0}=\mathcal{F} \bigcap \mathbb{R}_{++}^{2 n} .
$$

It is known (see, for example, [7]) that if $\mathcal{F}^{0}$ is nonempty, then the nonlinear system,

$$
\begin{aligned}
x s & =\tau e \\
Q x+R s & =b
\end{aligned}
$$

has a unique positive solution for any $\tau>0$. The set of all such solutions defines the central path $\mathcal{C}$ of the HLCP, that is,

$$
\mathcal{C}=\left\{z \in \mathbb{R}_{++}^{2 n}: F_{\tau}(z)=0, \tau>0\right\},
$$

where

$$
F_{\tau}(z)=\left[\begin{array}{c}
x s-\tau e \\
Q x+R s-b
\end{array}\right] .
$$

If $F_{\tau}(z)=0$, then it is easy to see that $\tau=\mu(z)$, where $\mu(z)$ is given by (1.2). The wide neighborhood $\mathcal{N}_{\infty}^{-}(\alpha)$ is defined as

$$
\mathcal{N}_{\infty}^{-}(\alpha)=\left\{z \in \mathcal{F}^{0}: \delta_{\infty}^{-}(z) \leq \alpha\right\},
$$


where $0<\alpha<1$ is a given parameter and

$$
\delta_{\infty}^{-}(z):=\left\|\left[\frac{x s}{\mu(z)}-e\right]^{-}\right\|_{\infty}
$$

is a proximity measure of $z$ to the central path. Alternatively, if we denote

$$
\mathcal{D}(\beta)=\left\{z \in \mathcal{F}^{0}: x s \geq \beta \mu(z)\right\}
$$

then the neighborhood $\mathcal{N}_{\infty}^{-}(\alpha)$ can also be written as

$$
\mathcal{N}_{\infty}^{-}(\alpha)=\mathcal{D}(1-\alpha)
$$

It is well known (see, for example, the proof in [15]) that

$$
\lim _{\alpha \downarrow 0} \mathcal{N}_{\infty}^{-}(\alpha)=\lim _{\beta \uparrow 1} \mathcal{D}(\beta)=\mathcal{C}, \quad \lim _{\alpha \uparrow 1} \mathcal{N}_{\infty}^{-}(\alpha)=\lim _{\beta \downarrow 0} \mathcal{D}(\beta)=\mathcal{F} .
$$

3. A higher order corrector-predictor algorithm. The higher order corrector and predictor use higher derivatives of the central path. Given a point $z=$ $\lceil x, s\rfloor \in \mathcal{D}(\beta)$, we consider the curve given by an $m$ th order vector valued polynomial of the form

$$
z(\theta)=z+\sum_{i=1}^{m} w^{i} \theta^{i}
$$

where the vectors $w^{i}=\left\lceil u^{i}, v^{i}\right\rfloor$ are obtained as solutions of the following linear systems

$$
\begin{aligned}
& \left\{\begin{aligned}
s u^{1}+x v^{1} & =\gamma \mu e-(1+\epsilon) x s \\
Q u^{1}+R v^{1} & =0
\end{aligned}\right. \\
& \left\{\begin{aligned}
s u^{2}+x v^{2} & =\epsilon x s-u^{1} v^{1}, \\
Q u^{2}+R v^{2} & =0
\end{aligned}\right. \\
& \left\{\begin{aligned}
s u^{i}+x v^{i} & =-\sum_{j=1}^{i-1} u^{j} v^{i-j}, \quad i=3, \ldots, m . \\
Q u^{i}+R v^{i} & =0
\end{aligned}\right.
\end{aligned}
$$

In a corrector step we choose $\epsilon=0$ and $\gamma \in[\underline{\gamma}, \bar{\gamma}]$, where $0<\underline{\gamma}<\bar{\gamma}<1$ are given parameters, while in a predictor step we take

$$
\gamma=0, \quad \text { and } \epsilon=\left\{\begin{array}{ll}
0, & \text { if HLCP is nondegenerate } \\
1, & \text { if HLCP is degenerate }
\end{array} .\right.
$$

We note that in the corrector step, where we have $\epsilon=0, w^{1}$ is the affine scaling direction if $\gamma=0$, and the classical centering direction if $\gamma=1$. In system $(3.2), w^{1}$ is a convex combination of the affine scaling and the centering directions. The directions $w^{i}$ are related to the higher derivatives of the central path [19]. We note that the central path passing through $z$ is analytic in $\mu$ when HLCP is nondegenerate, and in $\sqrt{\mu}$ when HLCP is degenerate.

As the $m$ linear systems in (3.2) have the same left hand matrix, only one matrix factorization and $m$ backsolves are needed. Therefore it involves $O\left(n^{3}\right)+O\left(m n^{2}\right)$ 
arithmetic operations. We take $m=m_{c}$ in the corrector step, and $m=m_{p}$ in the predictor step. From (3.1) and (3.2) it follows that

$$
\begin{aligned}
& x(\theta) s(\theta)=(1-\theta)^{1+\epsilon} x s+\gamma \theta \mu e+\sum_{i=m+1}^{2 m} \theta^{i} h^{i}, \\
& \mu(\theta)=(1-\theta)^{1+\epsilon} \mu+\gamma \theta \mu+\sum_{i=m+1}^{2 m} \theta^{i}\left(e^{T} h^{i} / n\right), \\
& \text { where } h^{i}=\sum_{j=i-m}^{m} u^{j} v^{i-j} .
\end{aligned}
$$

In the development of our algorithm, we want to preserve positivity of each iterated point. We thus give an upper bound $\theta_{0}$ for the step length taken both in the predictor and the corrector step:

$$
\theta_{0}=\sup \left\{\hat{\theta}_{0}: x(\theta)>0, s(\theta)>0, \forall \theta \in\left[0, \hat{\theta}_{0}\right]\right\} .
$$

We introduce the following notation, which will be used in describing both the corrector and predictor steps:

$$
p(\theta)=\frac{x(\theta) s(\theta)}{\mu(\theta)}, \quad f(\theta)=\min _{i=1, \ldots, n} p_{i}(\theta) .
$$

The corrector. The corrector step is obtained by taking $\epsilon=0$, and $0<\gamma<1$ in (3.1)-(3.2). The main purpose of the corrector step is to increase proximity to the central path. However, we also improve the normalized complementarity gap $\mu(\theta)$ at the same time. We choose $\sigma \in[\underline{\sigma}, \bar{\sigma}]$, where $0<\underline{\sigma}<\bar{\sigma}<1$ are given parameters, and define

$$
\theta_{1}=\sup \left\{\hat{\theta}_{1}: 0 \leq \hat{\theta}_{1} \leq \theta_{0}, \mu(\theta) \leq(1-\sigma(1-\gamma) \theta) \mu, \forall \theta \in\left[0, \hat{\theta}_{1}\right]\right\} .
$$

The step-length of the corrector is obtained as

$$
\theta_{c}=\operatorname{argmax}\left\{f(\theta): \theta \in\left[0, \theta_{1}\right]\right\} .
$$

As a result of the corrector step we obtain the point

$$
\bar{z}=\lceil\bar{x}, \bar{s}\rfloor:=z\left(\theta_{c}\right) .
$$

We have clearly $\bar{z} \in \mathcal{D}\left(\beta_{c}\right)$ with $\beta_{c}>\beta$. While the parameter $\beta$ is fixed during the algorithm, the positive quantity $\beta_{c}$ varies from iteration to iteration. However, we will prove that there is a constant $\beta_{c}^{*}>\beta$, such that $\beta_{c}>\beta_{c}^{*}$ in all iterations.

The predictor. The predictor is obtained by taking $z=\bar{z}$, where $\bar{z}$ is the result of the corrector step, and $\gamma=0$ in (3.1)-(3.2). The aim of the predictor step is to decrease the normalized complementarity gap as much as possible while keeping the iterate in $\mathcal{D}(\beta)$. We define the predictor step length as

$$
\theta_{p}=\operatorname{argmin}\left\{\mu(\theta): \theta \in\left[0, \theta_{2}\right]\right\},
$$

where

$$
\theta_{2}=\max \left\{\hat{\theta}_{2}: z(\theta) \in \mathcal{D}(\beta), \forall \theta \in\left[0, \hat{\theta}_{2}\right]\right\}
$$


A standard continuity argument can be used to show that $z(\theta)>0, \forall \theta \in\left[0, \theta_{2}\right]$. As a result of the predictor step, we obtain a point

$$
z^{+}=\left\lceil x^{+}, s^{+}\right\rfloor:=z\left(\theta_{p}\right) .
$$

By construction we have $z^{+} \in \mathcal{D}(\beta)$, so that a new corrector step can be applied. Summing up we can formulate the following iterative procedure:

Algorithm 1

Given real parameters $0<\beta<1,0<\underline{\gamma}<\bar{\gamma}<1,0<\underline{\sigma}<\bar{\sigma}<1$, integers $m_{c}, m_{p} \geq 1$, and a vector $z^{0} \in \mathcal{D}(\beta)$ :

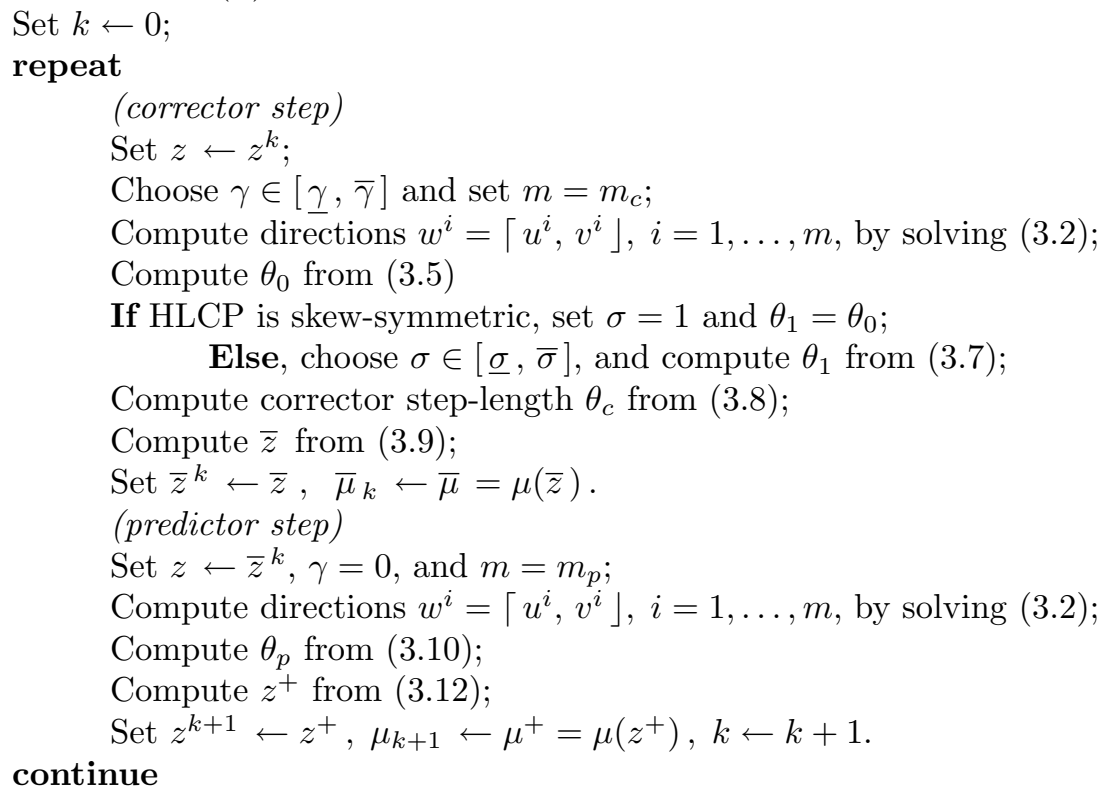

The computation of the exact values of $\theta_{c}$ and $\theta_{p}$ is quite involved, so that in practice good estimates of $\theta_{c}$ and $\theta_{p}$ are obtained by appropriate line search procedures. In particularly, by adopting the line search procedure from [15] we can preserve both the computational complexity and superlinear convergence of the theoretical algorithm. In fact the convergence properties can be proved by using the explicit lower bounds in the next section.

4. Polynomial complexity. We analyze in this section the computational complexity of Algorithm 1. In the proof of the complexity results, we will use the following lemmas, which were proved in [16].

LEMMA 4.1. Assume that HLCP (2.1) is $P_{*}(\kappa)$, and let $w=\lceil u, v\rfloor$ be the solution of the following linear system

$$
\begin{aligned}
s u+x v & =a \\
Q u+R v & =0
\end{aligned}
$$

where $z=\lceil x, s\rfloor \in \mathbb{R}_{++}^{2 n}$ and $a \in \mathbb{R}^{n}$ are given vectors, and consider the index sets:

$$
\mathcal{I}^{+}=\left\{i: u_{i} v_{i}>0\right\}, \quad \mathcal{I}^{-}=\left\{i: u_{i} v_{i}<0\right\} .
$$

Then the following inequalities are satisfied:

$$
\frac{1}{1+4 \kappa}\|u v\|_{\infty} \leq \sum_{i \in \mathcal{I}_{+}} u_{i} v_{i} \leq \frac{1}{4}\left\|(x s)^{-1 / 2} a\right\|_{2}^{2} .
$$


Lemma 4.2. Assume that HLCP (2.1) is $P_{*}(\kappa)$, and let $w=\lceil u, v\rfloor$ be the solution of the following linear system

$$
\begin{aligned}
s u+x v & =a \\
Q u+R v & =0
\end{aligned}
$$

where $z=\lceil x, s\rfloor \in \mathbb{R}_{++}^{2 n}$ and $a \in \mathbb{R}^{n}$ are given vectors. Then the following inequality holds:

$$
u^{T} v \geq-\kappa\left\|(x s)^{-1 / 2} a\right\|_{2}^{2}
$$

Let us denote

$$
\eta_{i}=\left\|D u^{i}+D^{-1} v^{i}\right\|_{2} \text {, where } D=X^{-1 / 2} S^{1 / 2} .
$$

The following lemma is a slight improvement over the corresponding results in [16] and a generalization to sufficient HLCP of the corresponding results in [15].

LEMMA 4.3. If $H L C P$ (2.1) is sufficient and $z=\lceil x, s\rfloor \in \mathcal{D}(\beta)$, then for $n \geq 8$, the solution of (3.2) satisfy

(4.3) $\frac{1}{\sqrt{1+2 \kappa}} \sqrt{\left\|D u^{i}\right\|_{2}^{2}+\left\|D^{-1} v^{i}\right\|_{2}^{2}} \leq \eta_{i} \leq \frac{2}{1+2 \kappa} \alpha_{i} \sqrt{\beta \mu}\left(\frac{(1+2 \kappa) \tau}{4} \sqrt{n}\right)^{i}$,

where

$$
\tau=\frac{2 \sqrt{\beta(1+\epsilon-\gamma)^{2}+(1-\beta) \gamma^{2}}}{\beta},
$$

and the sequence

$$
\alpha_{i}=\frac{1}{i}\left(\begin{array}{c}
2 i-2 \\
i-1
\end{array}\right) \leq \frac{1}{i} 4^{i}
$$

is the solution of the following recurrence scheme

$$
\alpha_{1}=1, \quad \alpha_{i}=\sum_{j=1}^{i-1} \alpha_{j} \alpha_{i-j} .
$$

Proof. The first part of the inequality follows immediately, since by using (3.2) and Lemma 4.2 we have

$$
\begin{gathered}
\left\|D u^{i}+D^{-1} v^{i}\right\|_{2}^{2}=\left\|D u^{i}\right\|_{2}^{2}+2 u^{i T} v^{i}+\left\|D^{-1} v^{i}\right\|_{2}^{2} \\
\geq\left\|D u^{i}\right\|_{2}^{2}+\left\|D^{-1} v^{i}\right\|_{2}^{2}-2 \kappa\left\|D u^{i}+D^{-1} v^{i}\right\|_{2}^{2} .
\end{gathered}
$$

By multiplying the first equations of $(3.2)$ with $(x s)^{-1 / 2}$ we obtain

$$
\begin{aligned}
D u^{1}+D^{-1} v^{1} & =-\left((1+\epsilon)(x s)^{1 / 2}-\gamma \mu(x s)^{-1 / 2}\right) \\
D u^{2}+D^{-1} v^{2} & =-\left(\epsilon(x s)^{1 / 2}-(x s)^{-1 / 2} u^{1} v^{1}\right) \\
D u^{i}+D^{-1} v^{i} & =-(x s)^{-1 / 2} \sum_{j=1}^{i-1} D u^{j} D^{-1} v^{i-j}, \quad 3 \leq i \leq m .
\end{aligned}
$$


Because $z \in \mathcal{D}(\beta)$ we have $(x s)^{-1 / 2} \leq(1 / \sqrt{\beta \mu}) e$, and we deduce that

$$
\eta_{1}=\left\|(1+\epsilon)(x s)^{1 / 2}-\gamma \mu(x s)^{-1 / 2}\right\|, \eta_{2}=\left\|\epsilon(x s)^{1 / 2}-(x s)^{-1 / 2} u^{1} v^{1}\right\|,
$$

and

$$
\eta_{i} \leq \frac{1}{\sqrt{\beta \mu}} \sum_{j=1}^{i-1}\left\|D u^{j}\right\|_{2}\left\|D^{-1} v^{i-j}\right\|_{2}, 3 \leq i \leq m .
$$

We have

$$
\begin{aligned}
\eta_{1}^{2} & =\left\|(1+\epsilon)(x s)^{1 / 2}-\gamma \mu(x s)^{-1 / 2}\right\|^{2}=\sum_{j=1}^{n}\left((1+\epsilon)^{2} x_{j} s_{j}-2(1+\epsilon) \gamma \mu+\frac{\gamma^{2} \mu^{2}}{x_{j} s_{j}}\right) \\
& =\left((1+\epsilon)^{2}-2(1+\epsilon) \gamma\right) \mu n+\gamma^{2} \mu^{2} \sum_{j=1}^{n} \frac{1}{x_{j} s_{j}} \\
& \leq \mu n\left((1+\epsilon)^{2}-2(1+\epsilon) \gamma+\frac{\gamma^{2}}{\beta}\right)=\frac{\beta \mu n \tau^{2}}{4},
\end{aligned}
$$

which shows that the second inequality in (4.3) is satisfied for $i=1$. We next show that the inequality also holds for $i=2$, i.e., we want to prove that

$$
\eta_{2}^{2} \leq\left(\frac{1+2 \kappa}{8}\right)^{2} \beta \mu n^{2} \tau^{4}=\frac{1}{128} \beta \mu n^{2} \tau^{4}\left(2+8 \kappa+8 \kappa^{2}\right) .
$$

Using Lemma4.2, Corollary 2.3 of [13], and the fact $z \in \mathcal{D}(\beta)$, we have

$$
\begin{aligned}
\eta_{2}^{2} & =\left\|\epsilon(x s)^{1 / 2}-(x s)^{-1 / 2} u^{1} v^{1}\right\|^{2}=\sum_{j=1}^{n}\left(\epsilon^{2} x_{j} s_{j}-2 \epsilon u_{i}^{1} v_{i}^{1}+\frac{\left(u_{i}^{1} v_{i}^{1}\right)^{2}}{x_{j} s_{j}}\right) \\
& \leq \epsilon^{2} n \mu+2 \epsilon \kappa \eta_{1}^{2}+\frac{\eta_{1}^{4}}{8 \beta \mu}\left(1+4 \kappa+8 \kappa^{2}\right) \leq \epsilon^{2} n \mu+\frac{\epsilon \kappa \beta \mu n \tau^{2}}{2}+\frac{\beta \mu n^{2} \tau^{4}}{128}\left(1+4 \kappa+8 \kappa^{2}\right) .
\end{aligned}
$$

Therefore, it remains to show that

$$
\epsilon^{2} n \mu+\frac{\epsilon \kappa \beta \mu n \tau^{2}}{2} \leq \frac{\beta \mu n^{2} \tau^{4}}{128}(1+4 \kappa),
$$

which holds trivially for $\epsilon=0$. The inequality holds for $\epsilon=1$ provided

$$
\beta n \tau^{4} \geq 128, \quad n \tau^{2} \geq 16 .
$$

Using the definition of $\tau$ (4.4), this reduces to

$$
\frac{n\left(\beta(2-\gamma)^{2}+(1-\beta) \gamma^{2}\right)^{2}}{\beta^{3}} \geq 8, \quad \frac{n\left(\beta(2-\gamma)^{2}+(1-\beta) \gamma^{2}\right)}{\beta^{2}} \geq 4 .
$$

Since the minimum over $0 \leq \beta, \gamma \leq 1$ of both left hand side functions is attained at $\beta=\gamma=1$, we conclude that that the second inequality in (4.3) is satisfied for $i=2$ whenever $n \geq 8$. For $i \geq 3$ and $1 \leq j<i$ we use the first inequality in (4.3) we obtain

$$
\begin{aligned}
& \left\|D u^{j}\right\|_{2}\left\|D^{-1} v^{i-j}\right\|_{2}+\left\|D u^{i-j}\right\|_{2}\left\|D^{-1} v^{j}\right\|_{2} \\
\leq & \left(\left\|D u^{j}\right\|_{2}^{2}+\left\|D^{-1} v^{j}\right\|_{2}^{2}\right)^{1 / 2}\left(\left\|D u^{i-j}\right\|_{2}^{2}+\left\|D^{-1} v^{i-j}\right\|_{2}^{2}\right)^{1 / 2} \\
\leq & (1+2 \kappa) \eta_{j} \eta_{i-j} .
\end{aligned}
$$


From (4.5) it follows that

$$
\eta_{i} \leq \frac{1+2 \kappa}{2 \sqrt{\beta \mu}} \sum_{j=1}^{i-1} \eta_{j} \eta_{i-j}, \quad i=2, \ldots, m .
$$

The required inequalities are then easily proved by mathematical induction.

By virtue of Lemma 4.3 we obtain the following bound for $\left\|h^{i}\right\|$.

LEMMA 4.4. If HLCP (2.1) is sufficient and $z=\lceil x, s\rfloor \in \mathcal{D}(\beta)$ then for $n \geq 8$, the directions computed in (3.4) satisfy

$$
\zeta_{i}:=\left\|h^{i}\right\| \leq \frac{2 \beta \mu}{(1+2 \kappa) i}((1+2 \kappa) \tau \sqrt{n})^{i}, i=m+1, \ldots, 2 m
$$

Proof. For any $m+1 \leq i \leq 2 m$, we have

$$
\begin{aligned}
\left\|h^{i}\right\|_{2} & \leq \sum_{j=i-m}^{m}\left\|D u^{j}\right\|_{2}\left\|D^{-1} v^{i-j}\right\|_{2} \leq \sum_{j=1}^{i-1}\left\|D u^{j}\right\|_{2}\left\|D^{-1} v^{i-j}\right\|_{2} \\
& =\frac{1}{2} \sum_{j=1}^{i-1}\left(\left\|D u^{j}\right\|_{2}\left\|D^{-1} v^{i-j}\right\|_{2}+\left\|D u^{i-j}\right\|_{2}\left\|D^{-1} v^{j}\right\|_{2}\right) \\
& \leq \frac{1}{2} \sum_{j=1}^{i-1} \sqrt{\left\|D u^{j}\right\|_{2}^{2}+\left\|D^{-1} v^{j}\right\|_{2}^{2}} \sqrt{\left\|D u^{i-j}\right\|_{2}^{2}+\left\|D^{-1} v^{i-j}\right\|_{2}^{2}} \\
& \leq \frac{1+2 \kappa}{2} \sum_{j=1}^{i-1} \eta_{j} \eta_{i-j} \leq \frac{2 \beta \mu}{1+2 \kappa}\left(\frac{(1+2 \kappa) \tau \sqrt{n}}{4}\right)^{i} \sum_{j=1}^{i-1} \alpha_{j} \alpha_{i-j} \\
& =\frac{2 \beta \mu}{1+2 \kappa}\left(\frac{(1+2 \kappa) \tau \sqrt{n}}{4}\right)^{i} \alpha_{i} \leq \frac{2 \beta \mu}{(1+2 \kappa) i}((1+2 \kappa) \tau \sqrt{n})^{i}
\end{aligned}
$$

where the last inequality follows from the fact that $\alpha_{i} \leq \frac{1}{i} 4^{i}$.

From the above lemmas we obtain the following result.

COROLlary 4.5. If HLCP (2.1) is sufficient and $z=\lceil x, s\rfloor \in \mathcal{D}(\beta)$ then the following relations hold for any $\alpha>0, \kappa \geq 0$, and $n \geq 8$ :

$$
\begin{aligned}
& (4.7) \frac{\alpha}{\mu} \sum_{i=m+1}^{2 m} \theta^{i}\left\|h^{i}\right\|_{2}<1, \forall 0 \leq \theta \leq \frac{1}{(1+2 \kappa) \tau \sqrt{n}} \min \left\{1,\left(\frac{1.4 \alpha \beta}{1+2 \kappa}\right)^{\frac{-1}{m+1}}\right\}, \\
& \text { (4.8) } \frac{\alpha}{\mu \sqrt{n}} \sum_{i=m+1}^{2 m} \theta^{i}\left\|h^{i}\right\|_{2}<\theta, \forall 0 \leq \theta \leq \frac{1}{(1+2 \kappa) \tau \sqrt{n}} \min \left\{1,(1.4 \alpha \beta \tau)^{\frac{-1}{m}}\right\},
\end{aligned}
$$

Proof. For any $t \in(0,1]$ we have

$$
\sum_{i=m+1}^{2 m} \frac{t^{i}}{i} \leq t^{m+1} \sum_{i=m+1}^{2 m} \frac{1}{i}<t^{m+1} \int_{m}^{2 m} \frac{d u}{u}=t^{m+1} \log 2<.7 t^{m+1} .
$$

Using Lemma 4.4 and the above inequality we obtain

(4.9) $\sum_{i=m+1}^{2 m} \theta^{i}\left\|h^{i}\right\|_{2}<\frac{1.4 \beta \mu}{1+2 \kappa}((1+2 \kappa) \tau \sqrt{n} \theta)^{m+1}, \quad \forall \theta \in\left(0, \frac{1}{(1+2 \kappa) \tau \sqrt{n}}\right]$. 
Therefore,

$$
\begin{aligned}
& \frac{\alpha}{\mu} \sum_{i=m+1}^{2 m} \theta^{i}\left\|h^{i}\right\|_{2}<\frac{1.4 \alpha \beta}{1+2 \kappa}((1+2 \kappa) \tau \sqrt{n} \theta)^{m+1} \leq 1, \\
& \forall \theta \in\left(0, \frac{1}{(1+2 \kappa) \tau \sqrt{n}} \min \left\{1,\left(\frac{1.4 \alpha \beta}{1+2 \kappa}\right)^{\frac{-1}{m+1}}\right\}\right] .
\end{aligned}
$$

(4.9) also implies that

$$
\begin{aligned}
\frac{\alpha}{\mu \sqrt{n}} \sum_{i=m+1}^{2 m} \theta^{i}\left\|h^{i}\right\|_{2}< & \frac{1.4 \alpha \beta}{(1+2 \kappa) \sqrt{n}}((1+2 \kappa) \tau \sqrt{n} \theta)^{m+1} \\
= & 1.4 \alpha \beta \tau \theta((1+2 \kappa) \tau \sqrt{n} \theta)^{m} \leq \theta \\
& \forall \theta \in\left(0, \frac{1}{(1+2 \kappa) \tau \sqrt{n}} \min \left\{1,(1.4 \alpha \beta \tau)^{\frac{-1}{m}}\right\}\right] .
\end{aligned}
$$

From the definition of $\tau$ (4.4) it follows that

$$
\frac{2(1+\epsilon) \sqrt{1-\beta}}{\sqrt{\beta}} \leq \tau \leq 2 \max \left\{\frac{1+\epsilon}{\sqrt{\beta}}, \frac{\sqrt{1-\beta+\beta \epsilon^{2}}}{\beta}\right\}<\frac{2(1+\epsilon)}{\beta} .
$$

In the corrector step we take $\epsilon=0$, therefore we will use the bound $\tau<2 / \beta$ in the analysis below.

THEOREM 4.6. If HCLP (2.1) is sufficient, then Algorithm 1 is well defined and the following relations hold for any $\kappa \geq 0$ and $n \geq 8$ :

$$
\begin{gathered}
\bar{z}^{k}, z^{k} \in \mathcal{D}(\beta), \\
\mu_{k+1} \leq\left(1-\frac{\chi}{(1+2 \kappa) n^{\frac{1}{2}+\frac{m_{c}+1}{2 m_{c}\left(m_{p}+1\right)}}}\right) \bar{\mu}_{k}, \quad k=0,1, \ldots, \\
\bar{\mu}_{k+1} \leq\left(1-\frac{\bar{\chi}}{(1+2 \kappa) n^{\frac{1}{2}+v}}\right) \bar{\mu}_{k}, \quad k=0,1, \ldots,
\end{gathered}
$$

where $\chi, \bar{\chi}$ are constants depending only on $\beta, \underline{\gamma}, \bar{\gamma}, \underline{\sigma}, \bar{\sigma}$, and

$$
v:=\min \left\{\frac{1}{2 m_{c}}, \frac{m_{c}+1}{2 m_{c}\left(m_{p}+1\right)}\right\} .
$$

Proof. Analysis of the corrector. On the corrector we have $m=m_{c}, \epsilon=0$, $0<\gamma<\gamma<\bar{\gamma}<1,0<\underline{\sigma}<\gamma<\bar{\sigma}<1$, and $\tau<2 / \beta$.

$\bar{F}$ irst, we prove that if $z \in \mathcal{D}(\beta)$, then the quantities $\theta_{0}$ defined in (3.5) satisfy

$$
\theta_{0} \geq \theta_{3}:=\frac{\beta}{2(1+2 \kappa) \sqrt{n}}\left(\frac{2.8}{1+2 \kappa}\right)^{-\frac{1}{m_{c}+1}}
$$


This can be shown by using (4.7) with $\alpha=2 / \beta$, and the fact that $\theta_{3}<1 / 2$,

$$
\frac{x(\theta) s(\theta)}{\mu}>(1-\theta) \frac{x s}{\mu}+\frac{1}{\mu} \sum_{i=m+1}^{2 m} \theta^{i} h^{i} \geq \frac{\beta}{2} e-\frac{1}{\mu} \sum_{i=m+1}^{2 m} \theta^{i}\left\|h^{i}\right\|_{2} e>0, \forall \theta \in\left[0, \theta_{3}\right] .
$$

Since $x(0)>0, s(0)>0$, we can use a standard continuity argument to show that $x(\theta)>0, s(\theta)>0, \forall \theta \in\left[0, \theta_{3}\right]$, which proves that $\theta_{0} \geq \theta_{3}$.

Next we show that the quantities $\theta_{1}$ defined in (3.7), satisfy

$$
\theta_{1} \geq \theta_{4}:=\frac{\beta}{2(1+2 \kappa) \sqrt{n}}\left(\frac{(1-\bar{\sigma})(1-\bar{\gamma})}{2.8}\right)^{\frac{1}{m_{c}}} .
$$

By using $(4.8)$ with $\alpha=1 /((1-\bar{\sigma})(1-\bar{\gamma}))$, we deduce that the following inequalities hold for any $\theta \in\left[0, \theta_{4}\right]$ :

$$
\begin{aligned}
& \frac{\mu(\theta)-(1-\sigma(1-\gamma) \theta) \mu}{\mu}=-(1-\sigma)(1-\gamma) \theta+\frac{1}{\mu n} \sum_{i=m+1}^{2 m} \theta^{i} e^{T} h^{i} \\
\leq & -(1-\bar{\sigma})(1-\bar{\gamma}) \theta+\frac{1}{\mu \sqrt{n}} \sum_{i=m+1}^{2 m} \theta^{i}\left\|h^{i}\right\|_{2} \leq 0,
\end{aligned}
$$

which shows that $\theta_{1} \geq \theta_{4}$.

At last, we show that if $z \in \mathcal{D}(\beta)$, then

$$
f(\theta) \geq \beta+\frac{1}{2}(1-\beta) \gamma \theta \geq \beta+\frac{1}{2}(1-\beta) \underline{\gamma} \theta, \quad \forall \theta \in\left[0, \theta_{5}\right],
$$

where

$$
\begin{aligned}
& (4.15) \theta_{5}:=\min \left\{\theta_{4}, \frac{\beta}{2(1+2 \kappa) n^{\frac{1}{2}+\frac{1}{2 m_{c}}}}\left(\frac{(1-\beta) \underline{\gamma}}{5.6}\right)^{\frac{1}{m_{c}}}\right\} \geq \frac{\chi_{5}}{(1+2 \kappa) n^{\frac{1}{2}+\frac{1}{2 m_{c}}}}, \\
& (4.16) \chi_{5}:=\frac{\beta}{2} \min \left\{\frac{(1-\bar{\sigma})(1-\bar{\gamma})}{2.8}, \frac{(1-\beta) \underline{\gamma}}{5.6}\right\} .
\end{aligned}
$$

It is easily seen that

$$
\begin{aligned}
p(\theta) & =\frac{x(\theta) s(\theta)}{\mu(\theta)}=\frac{(1-\theta) x s+\gamma \theta \mu e+\sum_{i=m+1}^{2 m} \theta^{i} h^{i}}{(1-\theta) \mu+\gamma \theta \mu+\sum_{i=m+1}^{2 m} \theta^{i} e^{T} h^{i} / n} \\
& \geq \frac{(1-\theta) \beta \mu e+\gamma \theta \mu e+\sum_{i=m+1}^{2 m} \theta^{i} h^{i}}{(1-\theta) \mu+\gamma \theta \mu+\sum_{i=m+1}^{2 m} \theta^{i} e^{T} h^{i} / n} \\
& =\beta e \frac{(1-\beta) \gamma \theta \mu e+\beta \sum_{i=m+1}^{2 m} \theta^{i}\left(h^{i}-\left(e^{T} h^{i} / n\right) e\right)+(1-\beta) \sum_{i=m+1}^{2 m} \theta^{i} h^{i}}{(1-\theta) \mu+\gamma \theta \mu+\sum_{i=m+1}^{2 m} \theta^{i} e^{T} h^{i} / n} \\
& \geq \beta e+\frac{(1-\beta) \gamma \theta \mu-\sum_{i=m+1}^{2 m} \theta^{i}\left\|h^{i}\right\|_{2}}{\mu(\theta)} e \\
(4.17) & \geq \beta e+(1-\beta) \gamma \theta-\frac{1}{\mu} \sum_{i=m+1}^{2 m} \theta^{i}\left\|h^{i}\right\|_{2} e, \quad \forall \theta \in[0,1] .
\end{aligned}
$$


The last inequality follows from the fact that

$$
\mu(\theta) \leq(1-\sigma(1-\gamma) \theta) \mu \leq \mu, \forall \theta \in\left[0, \theta_{4}\right]
$$

According to (4.8), with $\alpha$ replace by $2 \sqrt{n} /((1-\beta) \gamma)$, and $\tau$ replaced by $2 / \beta$, we have

$$
\frac{1}{\mu} \sum_{i=m+1}^{2 m} \theta^{i}\left\|h^{i}\right\|_{2}<\frac{1}{2}(1-\beta) \gamma \theta, \quad \forall \theta \leq \frac{\beta}{2(1+2 \kappa) \sqrt{n}}\left(\frac{(1-\beta) \gamma}{5.6 \sqrt{n}}\right)^{\frac{1}{m}} .
$$

and (4.14) follows from the above inequality and (4.17).

Relation (4.14) shows that if $z \in \mathcal{D}(\beta)$, then the point $\bar{z}$ obtained in the corrector step of Algorithm 1 belongs to $\mathcal{D}(\beta+\delta)$, where

$$
\delta=\frac{1}{2}(1-\beta) \gamma \theta_{5}
$$

As we mentioned before, the main purpose of the corrector is to increase proximity to the central path. However, it turns out that if the corrector step-length $\theta_{c}$ is large enough then we also obtain a significant reduction of the duality gap during the corrector step. In what follows we find a lower bound for $\theta_{c}$ in case the point $z \in \mathcal{D}(\beta)$ is not very well centered. More precisely we show that

$$
\exists j \quad \text { such that } p_{j}:=\frac{x_{j} s_{j}}{\mu} \leq \beta+.44 \delta \Rightarrow \theta_{c}>.2 \theta_{5} .
$$

Let us denote

$$
\lambda=.44 \delta=.22(1-\beta) \gamma \theta_{5}, q^{i}=\frac{h^{i}}{\mu}, i=m+1, \ldots, 2 m .
$$

For any $\theta \in[0,1]$ we have

$$
\begin{aligned}
p_{j}(\theta) & =\frac{x_{j}(\theta) s_{j}(\theta)}{\mu(\theta)}=\frac{(1-\theta) p_{j}+\gamma \theta+\sum_{i=m+1}^{2 m} \theta^{i} q_{j}^{i}}{(1-\theta)+\gamma \theta+\sum_{i=m+1}^{2 m} \theta^{i} e^{T} q^{i} / n} \\
& <\frac{(1-\theta)(\beta+\lambda)+\gamma \theta+\sum_{i=m+1}^{2 m} \theta^{i} q_{j}^{i}}{(1-\theta)+\gamma \theta+\sum_{i=m+1}^{2 m} \theta^{i} e^{T} q^{i} / n} \\
& =\beta+\lambda+\frac{\gamma(1-\beta-\lambda) \theta-(\beta+\lambda) \sum_{i=m+1}^{2 m} \theta^{i} e^{T} q^{i} / n+\sum_{i=m+1}^{2 m} \theta^{i} q_{j}^{i}}{1-(1-\gamma) \theta+\sum_{i=m+1}^{2 m} \theta^{i} e^{T} q^{i} / n} \\
& \leq \beta+\lambda+\frac{\gamma(1-\beta-\lambda) \theta+\left(1+\frac{\beta+\lambda}{\sqrt{n}}\right) \sum_{i=m+1}^{2 m} \theta^{i}\left\|q^{i}\right\|_{2}}{1-(1-\gamma) \theta-\frac{1}{\sqrt{n}} \sum_{i=m+1}^{2 m} \theta^{i}\left\|q^{i}\right\|_{2}} \\
& \leq \beta+\lambda+\frac{\gamma(1-\beta) \theta+2 \sum_{i=m+1}^{2 m} \theta^{i}\left\|q^{i}\right\|_{2}}{1-(1-\gamma) \theta-\sum_{i=m+1}^{2 m} \theta^{i}\left\|q^{i}\right\|_{2}}
\end{aligned}
$$

Assume now that $\theta \in\left[0, .2 \theta_{5}\right]$ and set $\theta=.2 \phi$. Since $\phi \in\left[0, \theta_{5}\right]$, by virtue of (4.8), we can write

$$
\begin{aligned}
\sum_{i=m+1}^{2 m} \theta^{i}\left\|q^{i}\right\|_{2} & =\sum_{i=m+1}^{2 m} .2^{i} \phi^{i}\left\|q^{i}\right\|_{2} \leq .2^{m+1} \sum_{i=m+1}^{2 m} \phi^{i}\left\|q^{i}\right\|_{2} \\
& <\frac{.2^{m+1}}{2} \gamma(1-\beta) \phi=\frac{.2^{m}}{2} \gamma(1-\beta) \theta \leq .1 \gamma(1-\beta) \theta .
\end{aligned}
$$


Using the fact that $\theta_{5}<.5, \forall n \geq 1$, we obtain

$$
\begin{aligned}
p_{j}(\theta) & <\beta+\lambda+\frac{1.2 \gamma(1-\beta) \theta}{1-(1-\gamma+.1 \gamma(1-\beta)) \theta} \leq \beta+\lambda+\frac{1.2 \gamma(1-\beta) \theta}{1-\theta} \\
& <\beta+\lambda+1.4 \gamma(1-\beta) \theta \leq \beta+\lambda+.28 \gamma(1-\beta) \theta_{5}=\beta+\delta, \quad \forall \theta \in\left[0, .2 \theta_{5}\right] .
\end{aligned}
$$

It follows that $f\left(\theta_{c}\right) \geq \beta+\delta>\max _{0 \leq \theta \leq .2 \theta_{5}} f(\theta)$, wherefrom we deduce that $\theta_{c}>.2 \theta_{5}$.

Analysis of the predictor. In the predictor step we have $\gamma=0$ and $m=m_{p}$. From (4.4) it follows $\tau=2(1+\epsilon) / \sqrt{\beta} \leq 4 / \sqrt{\beta}$. Since the predictor step follows a corrector step, we have $z \in \mathcal{D}(\beta+\delta) \subset \mathcal{D}(\beta)$.

First, we study the behavior of the normalized duality gap in the predictor step. We start by proving that

$$
\begin{aligned}
(1-2.5 \theta) \mu \leq & \mu(\theta) \leq(1-.5 \theta) \mu \\
& \forall 0 \leq \theta \leq \theta_{6}:=\frac{\sqrt{\beta}}{4(1+2 \kappa) \sqrt{n}} \min \left\{1,(11.2 \sqrt{\beta})^{\frac{-1}{m_{p}}}\right\} .
\end{aligned}
$$

Due to the obvious fact that

$$
(1-2 \theta) \leq(1-\theta)^{2} \leq(1-\theta), \quad \forall \theta \in[0,1]
$$

we have

$$
(1-2 \theta) \mu+\sum_{i=m+1}^{2 m} \theta^{i}\left(e^{T} h^{i} / n\right) \leq \mu(\theta) \leq(1-\theta) \mu+\sum_{i=m+1}^{2 m} \theta^{i}\left(e^{T} h^{i} / n\right) .
$$

Using (4.8), with $\alpha=2$ and $\tau=4 / \sqrt{\beta}$, we obtain

$$
\left|\sum_{i=m+1}^{2 m} \theta^{i}\left(e^{T} h^{i} / n\right)\right| \leq \frac{1}{\sqrt{n}} \sum_{i=m+1}^{2 m} \theta^{i}\left\|h^{i}\right\|_{2}<.5 \theta \mu
$$

for all $\theta \in\left[0, \theta_{6}\right]$. Using Lemma 4.4 and the sum of a geometric series with ratio .1, we deduce that for any $\theta \in\left[0, \frac{\sqrt{\beta}}{40(1+2 \kappa) \sqrt{n}}\right]$ holds

$$
\begin{aligned}
& \mu^{\prime}(\theta)=-(1+\epsilon-2 \epsilon \theta) \mu+\sum_{i=m+1}^{2 m} i \theta^{i-1}\left(e^{T} h^{i} / n\right) \leq-\mu+\frac{1}{\sqrt{n}} \sum_{i=m+1}^{2 m} i \theta^{i-1}\left\|h^{i}\right\|_{2} \\
& \leq-\mu+8 \mu \sqrt{\beta} \sum_{i=m}^{2 m-1}\left(\frac{4(1+2 \kappa) \theta \sqrt{n}}{\sqrt{\beta}}\right)^{i}<-\mu+8 \mu \sqrt{\beta} \frac{.^{m}}{1-.1}<-\mu+8 \mu \frac{1^{m}}{.9}<0 .
\end{aligned}
$$

Since $\theta_{6}>\frac{\sqrt{\beta}}{44.8(1+2 \kappa) \sqrt{n}}>\frac{\sqrt{\beta}}{50(1+2 \kappa) \sqrt{n}}$, we conclude that

$$
\begin{array}{r}
(1-2.5 \theta) \mu \leq \mu(\theta) \leq(1-.5 \theta) \mu \quad \text { and } \quad \mu^{\prime}(\theta)<0 \\
\forall \theta \in\left[0, \frac{\sqrt{\beta}}{50(1+2 \kappa) \sqrt{n}}\right] .
\end{array}
$$

Next, we claim that the quantity $\theta_{2}$ from (3.11) used in the computation of the predictor step-length satisfies 


$$
\begin{aligned}
\theta_{2} \geq \theta_{7} & :=\frac{\sqrt{\beta}}{4(1+2 \kappa) \sqrt{n}} \min \left\{1,(11.2 \sqrt{\beta})^{\frac{-1}{m_{p}}},\left(\frac{(1+2 \kappa) \delta}{2 \beta}\right)^{\frac{1}{m_{p}+1}}\right\} \\
& \geq \frac{\chi_{7}}{(1+2 \kappa) n^{\frac{1}{2}+\frac{m_{c}+1}{2 m_{c}\left(m_{p}+1\right)}}}, \\
\chi_{7} & :=\frac{1}{4} \min \left\{\frac{1}{11.2},\left(\frac{(1-\beta) \underline{\gamma} \chi_{5}}{4}\right)^{\frac{1}{2}}\right\} .
\end{aligned}
$$

Using (4.20) with $n \geq 8$ we obtain

$$
\mu(\theta) \geq\left(1-2.5 \theta_{6}\right) \mu \geq\left(1-\frac{2.5}{8 \sqrt{2}}\right) \mu \geq .7 \mu, \forall \theta \in\left[0, \theta_{6}\right] .
$$

By taking $\gamma=0$, and $\beta+\delta$ instead of $\beta$, in (4.17), using (4.7) with $\alpha=1 /(.7 \delta)$, we deduce that

$$
f(\theta) \geq \beta+\delta-\frac{\sum_{i=m+1}^{2 m} \theta^{i}\left\|h^{i}\right\|_{2}}{\mu(\theta)} \geq \beta+\delta-\frac{1}{.7 \mu} \sum_{i=m+1}^{2 m} \theta^{i}\left\|h^{i}\right\|_{2} \geq \beta, \forall \theta \in\left[0, \theta_{7}\right],
$$

which proves that $\theta_{2} \geq \theta_{7}$. From the definition of $\theta_{7}$ it follows

$$
\begin{aligned}
\theta_{7} & \geq \frac{\sqrt{\beta}}{4(1+2 \kappa) \sqrt{n}} \min \left\{\frac{1}{11.2 \sqrt{\beta}}, \frac{1}{\sqrt{\beta}}\left(\frac{(1+2 \kappa) \delta}{2}\right)^{\frac{1}{m_{p}+1}}\right\} \\
& \geq \frac{1}{4(1+2 \kappa) \sqrt{n}} \min \left\{\frac{1}{11.2},\left(\frac{(1-\beta) \underline{\gamma} \theta_{5}}{4}\right)^{\frac{1}{m_{p}+1}}\right\} \\
& \geq \frac{\sqrt{\beta}}{4(1+2 \kappa) \sqrt{n}} \min \left\{\frac{1}{11.2},\left(\frac{(1-\beta) \underline{\gamma} \chi_{5}}{4 n^{\frac{m_{c}+1}{2 m_{c}}}}\right)^{\frac{1}{m_{p}+1}}\right\} \\
& \geq \frac{\chi_{7}}{(1+2 \kappa) n^{\frac{1}{2}+\frac{m_{c}+1}{2 m_{c}\left(m_{p}+1\right)}}} \cdot
\end{aligned}
$$

Bounding the decrease of the duality gap. Due to the fact that the duality gap decreases in both predictor step and corrector step, a complete analysis of the decreases of the duality gap has to be done by studying a succession of correctorpredictor-corrector steps. Assume that we are at iteration $k$ and we have a point $z^{k} \in \mathcal{D}(\beta)$, with normalized duality gap $\mu_{k}$. We follow the notations of Algorithm 1 . The corrector step produces a point $\bar{z}^{k} \in \mathcal{D}(\beta+\delta)$, with $\delta$ given by (4.18). The corresponding normalized duality gap clearly satisfies $\bar{\mu}_{k} \leq \mu_{k}$, but a bound on the decrease of the duality gap cannot be given at this stage. The corrector is followed by a predictor that produces a point $z^{k+1}=z\left(\theta_{p}\right) \in \mathcal{D}(\beta)$ with duality gap $\mu_{k+1}=$ $\mu\left(\theta_{p}\right)=\min _{0 \leq \theta \leq \theta_{2}} \mu(\theta)$. We have $\theta_{7} \leq \theta_{2}$ and $\theta_{7} \leq \theta_{6}$, so that according to $(4.20)$

$$
\mu_{k+1} \leq \mu\left(\theta_{7}\right) \leq\left(1-.5 \theta_{7}\right) \bar{\mu}_{k} \leq\left(1-\frac{\chi_{7}}{2 n^{\frac{1}{2}+\frac{m_{c}+1}{2 m_{c}\left(m_{p}+1\right)}}}\right) \bar{\mu}_{k}, \quad \bar{\mu}_{k} \leq \mu_{k}
$$

The above relation is sufficient for proving polynomial complexity, but it does not take into account the contribution of the corrector step. A finer analysis is needed in order to account for that. We distinguish two cases: 
(a) $\theta_{2} \geq \frac{\sqrt{\beta}}{50(1+2 \kappa) \sqrt{n}}$. According to (4.21), in this case we have

$$
\mu_{k+1}=\min _{0 \leq \theta \leq \theta_{2}} \mu(\theta) \leq\left(1-\frac{\sqrt{\beta}}{100(1+2 \kappa) \sqrt{n}}\right) \bar{\mu}_{k} ;
$$

(b) $\theta_{2}<\frac{\sqrt{\beta}}{50(1+2 \kappa) \sqrt{n}}$. In this case $\mu(\theta)$ is decreasing on the interval $\left[0, \theta_{2}\right]$, by virtue of (4.21), and by using (4.23) we deduce that $\theta_{p}=\theta_{2}, f\left(\theta_{p}\right)=\beta$. The latter equality must be true, since if $f\left(\theta_{p}\right)>\beta$, then, by a continuity argument, it follows that $\theta_{2}>\theta_{p}$ which is a contradiction (see the definition of $\left.\theta_{2}(3.11)\right)$. But if $f\left(\theta_{p}\right)=\beta$, then, according to (3.8), in the next corrector step we have $\theta_{c}>.2 \theta_{5}$, so that

$$
\bar{\mu}_{k+1}<\left(1-.2 \underline{\sigma}(1-\bar{\gamma}) \theta_{5}\right) \mu_{k+1} \leq\left(1-\frac{\underline{\sigma}(1-\bar{\gamma}) \chi_{5}}{5(1+2 \kappa) n^{\frac{1}{2}+\frac{1}{2 m_{c}}}}\right) \mu_{k+1} .
$$

In conclusion, for any $k \geq 0$ we have

$$
\bar{\mu}_{k+1} \leq \mu_{k+1} \leq\left(1-\frac{\sqrt{\beta}}{100(1+2 \kappa) \sqrt{n}}\right) \bar{\mu}_{k}
$$

or

$$
\bar{\mu}_{k+1}<\left(1-\frac{\underline{\sigma}(1-\bar{\gamma}) \chi_{5}}{5(1+2 \kappa) n^{\frac{1}{2}+\frac{1}{2 m_{c}}}}\right)\left(1-\frac{\chi_{7}}{2(1+2 \kappa) n^{\frac{1}{2}+\frac{m_{c}+1}{2 m_{c}\left(m_{p}+1\right)}}}\right) \bar{\mu}_{k} .
$$

By taking

$$
\bar{\chi}:=\min \left\{\frac{\sqrt{\beta}}{100}, \frac{\sigma(1-\bar{\gamma}) \chi_{5}}{5}, \frac{\chi_{7}}{2}\right\}
$$

we deduce that

$$
\bar{\mu}_{k+1} \leq\left(1-\frac{\bar{\chi}}{(1+2 \kappa) n^{\frac{1}{2}+v}}\right) \bar{\mu}_{k}, \quad k=0,1, \ldots,
$$

where $v$ is given by (4.11). The proof is complete

As an immediate consequence of the above theorem we obtain the following complexity result:

COROLlary 4.7. Algorithm 1 produces a point $z=\lceil x, s\rfloor \in \mathcal{D}(\beta)$ with $x^{T} s \leq \varepsilon$, in at most $O\left((1+\kappa) n^{1 / 2+v} \log \left(x^{0 T} s^{0} / \varepsilon\right)\right)$ iterations, where $v$ is given by (4.11). It follows that if the order of either the corrector or the predictor is larger than a multiple of $\log n$, then Algorithm 1 has $O((1+\kappa) \sqrt{n} L)$-iteration complexity.

Corollary 4.8. If $\max \left\{m_{c}, m_{p}\right\}=\Omega(\log n)$, then Algorithm 1 produces a point $z=\lceil x, s\rfloor \in \mathcal{D}(\beta)$ with $x^{T} s \leq \varepsilon$, in at most $O\left((1+\kappa) \sqrt{n} \log \left(x^{0} s^{0} / \varepsilon\right)\right)$.

Proof. Under the hypothesis of the corollary there is a constant $\vartheta$, such that $v \leq \vartheta / \log n$. Hence $n^{1 / 2+v} \leq n^{\frac{\vartheta}{\log n}} \sqrt{n}=e^{\vartheta} \sqrt{n}$.

Due to the fact that $\lim _{n \rightarrow \infty} n^{1 / n^{\omega}}=1$ for any $\omega \in(0,1)$, in applications we can choose $m_{p}=\left\lceil n^{\omega}\right\rceil$ for some value of $\omega \in(0,1)$. This choice was initially suggested by Roos (private communication) and subsequently used in [24] and [16]. A correspondence between $n$ and $\left\lceil n^{\omega}\right\rceil$ with $\omega=0.1$ is shown in table 4 . 
TABLE 4.1

\begin{tabular}{crrrrrrr}
$n$ & $10^{4}$ & $10^{5}$ & $10^{6}$ & $10^{7}$ & $10^{8}$ & $10^{9}$ & $10^{10}$ \\
$\left\lceil n^{1}\right\rceil$ & 3 & 4 & 4 & 6 & 7 & 8 & 11 \\
\hline
\end{tabular}

5. Superlinear convergence. In this section we show that the duality gap of the sequence produced by Algorithm 1 is superlinearly convergent. The result is based on the following lemma which is a consequence of the results about the analyticity of the central path from [19].

LEMMA 5.1. If HLCP (2.1) is sufficient then the solution of (3.2) with $\gamma=0$ satisfies

$$
u^{i}=O\left(\mu^{i}\right), \quad v^{i}=O\left(\mu^{i}\right), \quad i=1, \ldots, m, \text { if } H L C P \text { (2.1) is nondegenerate, }
$$

and

$$
u^{i}=O\left(\mu^{i / 2}\right), \quad v^{i}=O\left(\mu^{i / 2}\right), \quad i=1, \ldots, m, \text { if } H L C P \text { (2.1) is degenerate } .
$$

By using the above lemma we obtain the following superlinear convergence result, which is a trivial extension of the corresponding result in [15] to sufficient linear complementarity problems.

TheOREM 5.2. The sequence $\mu_{k}$ produced by Algorithm 1 satisfies

$$
\mu_{k+1}=O\left(\mu_{k}^{m_{p}+1}\right) \text {, if } H L C P \text { (2.1) is nondegenerate, }
$$

and

$$
\mu_{k+1}=O\left(\mu_{k}^{\left(m_{p}+1\right) / 2}\right) \text {, if } H L C P(2.1) \text { is degenerate. }
$$

6. Conclusions. We have presented a corrector-predictor interior point algorithm for sufficient horizontal linear complementarity problems acting in a wide neighborhood of the central path.

The corrector of order $m_{c}$ is used to improve both the centrality and the complementarity gap. The predictor of order $m_{p}$ follows each corrector step to further decrease the complementarity gap. If $\max \left\{m_{c}, m_{p}\right\}=\Omega(\log n)$, then the iteration complexity of the algorithms is $O((1+\kappa) \sqrt{n} L)$. Although the complexity of our algorithm depends on $\kappa$, the algorithm itself does not, so that the algorithm works for the class of sufficient HLCP. Our algorithm has the best known iteration complexity for sufficient linear complementarity problems and is superlinearly convergent even for degenerate problems. The cost of implementing one iteration of our algorithm is $O\left(n^{3}\right)$ arithmetic operations.

\section{REFERENCES}

[1] M. Anitescu, G. Lesaja, and F. A. Potra. Equivalence between different formulations of the linear complementarity problem. Optimization Methods \&5 Software, 7(3):265-290, 1997. 
[2] K.M Anstreicher and R.A. Bosch. A new infinity-norm path following algorithm for linear programming. SIAM J. Optim., 5(2):236-246, 1995.

[3] R. W. Cottle, J.-S. Pang, and R. E. Stone. The Linear Complementarity Problem. Academic Press, Boston, MA, 1992.

[4] C. C. Gonzaga. Complexity of predictor-corrector algorithms for LCP based on a large neighborhood of the central path. SIAM J. Optim., 10(1):183-194 (electronic), 1999.

[5] P-F. Hung and Y. Ye. An asymptotical $(O \sqrt{n} L)$-iteration path-following linear programming algorithm that uses wide neighborhoods. SIAM Journal on Optimization, 6(3):159-195, August 1996.

[6] J. Ji, F. A. Potra, and S. Huang. A predictor-corrector method for linear complementarity problems with polynomial complexity and superlinear convergence. Journal of Optimization Theory and Applications, 84(1):187-199, 1995.

[7] M. Kojima, N. Megiddo, T. Noma, and A. Yoshise. A Unified Approach to Interior Point Algorithms for Linear Complementarity Problems, volume 538 of Lecture Notes in Comput. Sci. Springer-Verlag, New York, 1991.

[8] J. Miao. A quadratically convergent $O((1+k) \sqrt{n} L)$-iteration algorithm for the $P_{*}(k)$-matrix linear complementarity problem. Mathematical Programming, 69:355-368, 1995.

[9] S. Mizuno, M. J. Todd, and Y. Ye. On adaptive-step primal-dual interior-point algorithms for linear programming. Mathematics of Operations Research, 18(4):964-981, 1993.

[10] R. D. C. Monteiro and S. J. Wright. Local convergence of interior-point algorithms for degenerate monotone LCP. Computational Optimization and Applications, 3:131-155, 1994.

[11] R.C. Monteiro, I. Adler, and M.G. Resende. A polynomial-time primal-dual affine scalling algorithm for linear and convex quadratic programming and its power series extension. Mathematics of Operations Research, 15:191-214, 1990.

[12] J. Peng, T. Terlaky, and Y. B. Zhao. A self-regularity based predictor-corrector algorithm for linear optimization. Preprint submitted for publication, Department of Computing and Software, McMaster University, Hamilton, Ontario, Canada.

[13] F. A. Potra. An $O(n L)$ infeasible interior-point algorithm for LCP with quadratic convergence. Annals of Operations Research, 62:81-102, 1996.

[14] F. A. Potra. A superlinearly convergent predictor-corrector method for degenerate LCP in a wide neighborhood of the central path with $O(\sqrt{n} l)$-iteration complexity. Math. Programming, 100:317-337, 2004.

[15] F. A. Potra. Corrector-predictor methods for monotone linear complementarity problems in a wide neighborhood of the central path. preprint, UMBC, September 2004, Revised February 2006, Accepted by Mathematical Programming.

[16] F. A. Potra and X. Liu. Predictor-corrector methods for sufficient linear complementarity problems in a wide neighborhood of the central path. Optimization Methods and Software, 20(1):145-168, 2005.

[17] F. A. Potra and R. Sheng. A large-step infeasible-interior-point method for the $P_{*}$-matrix LCP. SIAM Journal on Optimization, 7(2):318-335, 1997.

[18] J. Stoer and M. Wechs. Infeasible-interior-point paths for sufficient linear complementarity problems and their analyticity. Math. Programming, 83(3, Ser. A):407-423, 1998.

[19] J. Stoer, M. Wechs, and S. Mizuno. High order infeasible-interior-point methods for solving sufficient linear complementarity problems. Math. Oper. Res., 23(4):832-862, 1998.

[20] Josef Stoer. High order long-step methods for solving linear complementarity problems. Ann. Oper. Res., 103:149-159, 2001. Optimization and numerical algebra (Nanjing, 1999).

[21] J. F. Sturm. Superlinear convergence of an algorithm for monotone linear complementarity problems, when no strictly complementary solution exists. Mathematics of Operations Research, 24:72-94, 1999.

[22] H. Väliaho. $P_{*}$-matrices are just sufficient. Linear Algebra and its Applications, 239:103-108, 1996.

[23] Y. Ye and K. Anstreicher. On quadratic and $O(\sqrt{n} L)$ convergence of predictor-corrector algorithm for LCP. Mathematical Programming, 62(3):537-551, 1993.

[24] G. Zhao. Interior point algorithms for linear complementarity problems based on large neighborhoods of the central path. SIAM J. Optim., 8(2):397-413 (electronic), 1998. 